\title{
Evolution of circadian organization in vertebrates
}

M. Menaker ${ }^{1}$, L.F. Moreira ${ }^{1,2}$ and G. Tosini ${ }^{1}$

\author{
${ }^{1} \mathrm{NSF}$ Center for Biological Timing and Department of Biology, University of Virginia, \\ Charlottesville, VA 22903, USA \\ ${ }^{2}$ Laboratório de Cronobiologia, Universidade Federal do Rio Grande do Norte, \\ 59072-970 Natal, RN, Brasil
}

\begin{abstract}
Correspondence
M. Menaker

Department of Biology

University of Virginia

Charlottesville, VA 22903

USA

Fax: (804) 982-5626

E-mail: mm7e@virginia.edu

Presented at the XI Annual Meeting of the Federação de Sociedades de Biologia Experimental, Caxambu, MG, Brasil, August 21-24, 1996.

Research supported by AFSOR (No. F49620-94-1-0356) and NIH (No. HD-13162) to M. Menaker.
\end{abstract}

Received December 2, 1996 Accepted December 20, 1996

\begin{abstract}
Circadian organization means the way in which the entire circadian system above the cellular level is put together physically and the principles and rules that determine the interactions among its component parts which produce overt rhythms of physiology and behavior. Understanding this organization and its evolution is of practical importance as well as of basic interest. The first major problem that we face is the difficulty of making sense of the apparently great diversity that we observe in circadian organization of diverse vertebrates. Some of this diversity falls neatly into place along phylogenetic lines leading to firm generalizations: i) in all vertebrates there is a "circadian axis" consisting of the retinas, the pineal gland and the suprachiasmatic nucleus (SCN), ii) in many non-mammalian vertebrates of all classes (but not in any mammals) the pineal gland is both a photoreceptor and a circadian oscillator, and iii) in all non-mammalian vertebrates (but not in any mammals) there are extraretinal (and extrapineal) circadian photoreceptors. An interesting explanation of some of these facts, especially the differences between mammals and other vertebrates, can be constructed on the assumption that early in their evolution mammals passed through a "nocturnal bottleneck". On the other hand, a good deal of the diversity among the circadian systems of vertebrates does not fall neatly into place along phylogenetic lines. In the present review we will consider how we might better understand such "phylogenetically incoherent" diversity and what sorts of new information may help to further our understanding of the evolution of circadian organization in vertebrates.
\end{abstract}

Key words

- Circadian rhythms

- Evolution

- Vertebrates

- Pineal

- SCN

- Retina

- Melatonin

Mer.
Organisms, from unicellulars to vertebrates, are structured in time as well as in space. Many, if not most, biochemical, physiological and behavioral parameters exhibited by organisms show daily fluctuations and most of these daily rhythms persist in constant conditions, thus demonstrating that they are driven by endogenous oscillators. The rhythms that persist in constant condi- tions with periods close to $24 \mathrm{~h}$ are called circadian rhythms.

By circadian organization we mean the way in which the entire circadian system above the cellular level is put together physically, and the principles and rules that determine the interactions among its component parts. Circadian organization extends both broadly and deeply into the physiology and 
behavior of multicellular organisms. At the core of the system that controls and regulates the many overt rhythms that can be measured in vertebrates are three structures that together with their interconnections form a central "circadian axis" common to all vertebrates, even the most primitive ones. These structures are the retinas, the pineal complex (pineal and parietal eye/organ) and the suprachiasmatic nucleus (SCN) of the hypothalamus. In one vertebrate species or another each of these structures has been shown to be involved in the control of circadian rhythmicity and/or to contain circadian oscillators capable of sustaining rhythmicity in vitro (Table 1).

Until recently the only cultured retinas that had been shown to oscillate with a circadian period in vitro were those of Xenopus (1) and chicks (2), however, at present we know that the retinas of some lampreys (3), lizards (4) and mammals (5) also have this property. The pineals of some fish, lizards and birds oscillate in culture while those of others do not $(6,7)$. No mammalian pineal has been shown to contain circadian oscillators although in intact mammals pineal rhythmicity driven from the $\mathrm{SCN}$ is robust (8). Only in mammals has the SCN been cultured and transplanted into lesioned animals and both kinds of experiments have provided conclusive evidence that it contains circadian oscillators (9). It is generally believed that the SCN (or its homolog) is intrinsically

Table 1 - Structures of the vertebrate brain in which the presence of circadian oscillators has been demonstrated by in vitro experiments (direct) or by lesion, transplant or other (indirect) techniques.

*Indicates classes and organs in which data on transplant are available.

\begin{tabular}{lll}
\hline & Direct & Indirect \\
\hline Cyclostome & retina, pineal & optic lobe, pineal \\
Fish & retina, pineal & pineal \\
Amphibian & retina & pineal \\
Reptile & retina, parietal eye, pineal & SCN, pineal \\
Bird $^{*}$ & retina, pineal & pineal*, lateral eyes, SCN \\
Mammal* $^{*}$ & retina, SCN & $\mathrm{SCN}^{*}$, lateral eyes
\end{tabular}

rhythmic in non-mammalian vertebrates, but this belief is based solely on lesioning experiments of various kinds which are in principle inadequate to establish the point firmly.

If one takes a broad view of vertebrate circadian systems, what emerges is a picture containing some common threads which only very roughly follow phylogenetic lines, overlain with a good deal of variability in the important details. It is not possible to say that all members of one vertebrate class are organized in a particular way (with the possible exception of the mammals to which we shall return). Indeed, among the reptiles not all lizards nor even all iguanid lizards have similarly organized circadian systems (10). Identifying the common threads and accounting for the variability are major challenges; until they have been met we will not fully understand the system we are studying nor be able to make the most productive use of the many experimental models with which natural selection has provided us.

Our hypothesis is that the vertebrate circadian axis is very old - indeed as old as the vertebrates themselves - and, in addition, that it is relatively "easy" to modify and has in fact been modified many times in the last 500 million years, each time in response to selection pressures in particular environmental niches. If that were all there is to it, the task of unraveling 500 million years of response to the many environments occupied by the vertebrates during that time would be hopeless. However, the task may not be hopeless because it seems likely that circadian systems have been shaped predominantly by one aspect of their environment, i.e., light. A corollary of our hypothesis therefore is that the differences in circadian organization that one finds among the vertebrates are to a large extent the consequence of rapid adaptation to particular photic niches into which groups have been pushed by a variety of unrelated selection pressures. If that were true then it should be possible to correlate particular aspects of circadian organization with the 
photic history of the groups in which they are found. The attempt to establish such correlations is made difficult by several uncertainties, among them are: i) it is hard to define the photic niche occupied even by living organisms much less those that have become extinct, ii) we don't know the photic histories of most organisms (i.e., the photic environments that they occupied during their evolution) in much detail, iii) we have no idea how quickly circadian organization can be modified by selection, and iv) there are undoubtedly other undefined pressures acting on the system at the same time as those from the photic environment. Nonetheless, some interesting correlations can be made and this fact alone encourages us to believe that our hypothesis may prove to be fruitful.

Let us first examine the proposition that the circadian axis is ancient by comparing the structure and function of this axis in a modern group, the passerine birds, to what we know about it in an ancient one, the cyclostomes. In passerines the dominant circadian oscillator is the pineal gland; removing it surgically renders the normal circadian locomotor behavior arrhythmic, while transplanting a donor pineal into the anterior chamber of the eye restores rhythmicity with the phase of the donor bird (11). In vitro, the pineal produces melatonin with a circadian rhythm and is photosensitive (12). Exogenous melatonin administered rhythmically restores locomotor rhythmicity to pinealectomized birds $(13,14)$. The presence of another circadian oscillator in the hypothalamus is suggested by the fact that lesions which include the SCN also make birds arrhythmic (15). Although there is no published work on retinal rhythmicity in passerines, the retinas of some other birds (chickens, quail and pigeons) appear to synthesize melatonin rhythmically and probably contain circadian oscillators (16).

Circadian rhythmicity has also been studied in the lamprey Lampetra japonica, a cyclostome. Adult lampreys on their way up river to spawn express clear free-running circadian rhythms of locomotor behavior when held under constant conditions in the laboratory (17). These rhythms are abolished by pinealectomy (17). In culture, the pineal of these lampreys produces melatonin with a circadian rhythm (18). Also, pineals and retinas from adults of a different species of lamprey (Petromyzon marinus) contain circadian oscillators that control rhythmic synthesis of melatonin (19). Although facts are missing that would complete the comparison (e.g., the results of pineal transplant experiments and the effects of rhythmically administered exogenous melatonin on the locomotor behavior of pinealectomized lampreys) there seems little doubt that in both birds and lampreys the photoreceptive pineal gland contains circadian oscillators that regulate the rhythmic production of the hormone melatonin which in turn may be responsible for the circadian aspect of locomotor behavior. It stretches (at least our) credulity to believe that these detailed similarities in biochemistry (melatonin), morphology and physiology (the pineal) and behavior (locomotion) have arisen by convergent evolution. Since the most recent common ancestor of cyclostomes and the rest of the vertebrates existed more than 450 million years ago we must conclude that the control of locomotor behavior by rhythmic pineal melatonin secretion is an ancient and primitive component of vertebrate circadian organization. Clearly, rhythmic synthesis of melatonin by the retina is equally old although we do not yet fully understand its function. These conclusions are not weakened by the fact that circadian organization in the other extant group of cyclostomes, the hagfish, is quite different, as will be discussed below.

Having arrived at some understanding of primitive vertebrate circadian systems, can we offer reasonable explanations for the variability that has arisen in the course of their evolution, based on the hypothesized impor- 
Table 2 - Distribution of circadian photoreceptors within the vertebrate central nervous system.

Extraretinal photoreceptors are present in all the vertebrate classes, except mammals. DBP, Deep brain photoreceptors.

\begin{tabular}{lcccc}
\hline & Pineal & $\begin{array}{c}\text { Parietal eye or } \\
\text { parapineal } \\
\text { organ }\end{array}$ & Retina & DBP \\
\hline Cyclostome & Yes & Yes & Yes & Yes \\
Fish & Yes & - & Yes & Yes \\
Amphibian & Yes & Yes & Yes & Yes \\
Reptile & Yes & Yes & Yes & Yes \\
Bird & Yes & - & Yes & Yes \\
Mammal & No & - & Yes & No
\end{tabular}

tance of photic history? One aspect of this variability is in the way in which different vertebrates perceive the light that affects their circadian systems (Table 2). With one possible exception (20) non-mammalian vertebrates have multiple circadian photoreceptors in retinas, pineal gland and still incompletely described "deep brain" photoreceptors (21). Mammals, on the other hand, use retinal photoreception exclusively (22). This broad dichotomy can be accounted for plausibly by the fact that early in their evolutionary history mammals were squeezed through what we have called elsewhere a "nocturnal bottleneck" (19). Perhaps aided by their newly acquired ability to thermoregulate physiologically, primitive mammals could best survive in a world in which the daylight hours were filled with dangerous reptilian predators by becoming nocturnal. Only later when the disappearance of many of the reptiles made the day relatively safe did mammals radiate into that temporal niche. Diurnal mammals are secondarily derived from nocturnal ancestors. Mammals are the only group of vertebrates that, as an entire class, has such a nocturnal heritage and we would argue that it caused the shift from multiple distributed circadian photoreceptors to a single site in the retina. To be convincing such an argument must address three distinct questions: i) What was the initial advantage of having multiple circadian photoreceptors, many of which (all?) were "non-visual", i.e., incapable of forming images? ii) Why did mammals retain those in the retina? iii) Why did they lose all the others?

The first of these is the most difficult. In thinking about the possible selective advantages of what at first seems a kind of photoreceptor extravagance it is helpful to remember that photoreceptors that inform their owners simply that light of a certain intensity is present or not, require only a light-absorbing molecule connected in some way to a membrane channel, and have arisen over and over again in almost every group of living things. A general explanation of the advantages obtained by having several circadian photoreceptors might fall into one or both of two categories: either each of the several photoreceptors might be tuned to respond to different aspects of the photic environment, or each photoreceptor may provide information about the photic environment to the specific tissue in which it resides. The second general category is the more appealing, especially when it is recognized that there is usually a circadian oscillator directly associated with each of the distributed circadian photoreceptors. Whatever the advantages of such organization may be they are certainly real, for the arrangement is widespread among the invertebrates as well (perhaps the most dramatic example being the presence of both a circadian oscillator and its photoreceptor in the testis of the gypsy moth (23)). However, there is at least one clear example of the first general category (i.e., specialized tuning of a vertebrate circadian photoreceptor in the unusual chromatic response of the lizard parietal eye (24)). That circadian photoreception may be incompatible with image formation has been convincingly argued by Cooper et al. (25) with respect to retinal circadian photoreceptors (about which more below) but the arguments apply equally well to extraretinal photoreceptors. Thus, multiple circadian photo- 
receptors may have been selected because they do not form images, can be specially tuned and can be located adjacent to the oscillators that require their input.

Such a system works well as long as there is enough light in the photic environment to reach and activate each of the multiple photoreceptors and it therefore presents no problems for diurnal organisms. Nocturnal animals, on the other hand, may be exposed only to very dim light at dusk and/or dawn. In that photic environment only the most sensitive photoreceptors in the most exposed location may receive enough light to guarantee a response. That consideration alone may explain why mammals came to depend on circadian photoreceptors in their retinas when they were forced into an exclusively nocturnal niche; but why lose all the extraretinal photoreceptors? The weak answer is that when a structure is no longer used it is lost because of the inefficiencies involved in maintaining it, but in this case there may be a stronger one. Of necessity multiple photoreceptors in different anatomical locations will receive different intensities of light and thus the same photic environment will stimulate some to a greater extent than others. As the light fades at dusk (or increases at dawn), intensities will occur that will stimulate only the most sensitive photoreceptors. Under these circumstances the organism with multiple photoreceptors runs the risk of receiving conflicting messages. One of its photoreceptors will tell it that it is still light while the others will signal darkness. Such conflicting signals would be clearly maladaptive in a system whose major selective advantage accrues from its ability to confer exquisite control of the phase relationships among its many circadian rhythms as well as the phase of the organism's activities relative to the environmental light cycle. It is not hard to imagine that the generation of inappropriate phase relationships as a consequence of conflicting signals from differentially illuminated circadian photoreceptors may have led, for example, to the loss of pineal photosensitivity in mammals which was achieved simply (and rapidly?) by eliminating the chromophore while leaving most of the rest of the photoreceptive machinery intact (26).

Circadian photoreceptors have been present in the vertebrate retina for 500 million years (19) but to depend on them exclusively may not have been tried before the early mammals became nocturnal (but see discussion of hagfish below). That may well have required some rewiring of the retinohypothalamic connection to achieve an appropriately non-visual circadian input and may have led to a more profound reorganization of the entire circadian system. With this accomplished the diurnal mammals that evolved subsequently would have been under no pressure to reinvent the distributed system of their non-mammalian ancestors.

If the arguments advanced above are generally correct one should see signs of similar changes in nocturnal non-mammalian vertebrates and perhaps among nocturnal invertebrates. The available data, although sparse, do support the general proposition that when animals become nocturnal they reduce the number of their circadian photoreceptors. Among the reptiles, geckos (nocturnal lizards) and snakes, most of which are nocturnal, lack parietal eyes and alligators do not have either parietal eyes or recognizable pineal glands (27). The most conspicuously nocturnal birds, the owls, either do not have pineal glands or those that they have are much reduced (28). Cockroaches, holometabolous insects which are nocturnal throughout their lives, have no extraretinal circadian photoreceptors (their ocelli have no input to the circadian system (29)) in contrast to many other insects which are diurnal at some stage in their development.

Another interesting example supporting our hypothesis is represented by the circadian organization of the hagfish, the only living cyclostomes besides lampreys (30). Their circadian systems are organized quite 
differently from the "primitive pattern" that characterizes lampreys and passerine birds. Uniquely among non-mammalian vertebrates, hagfish use only their eyes as circadian photoreceptors (20). They apparently have no pineal gland and the evidence, although still incomplete, suggests strongly that the circadian pacemaker that controls their surprisingly robust locomotor rhythm is located in the preoptic nucleus of the anterior hypothalamus (31). This pattern of circadian organization closely parallels that which we find in mammals and which we have attributed to their early forced nocturnality. Under laboratory conditions hagfish are nocturnal and one could argue that their nocturnality accounts for their mammal-like circadian organization, but the argument appears to fall apart when one recognizes that lampreys are also nocturnal both in the laboratory and in the field. There is, however, a big difference between the photic environments of lampreys and hagfish. As adults lampreys are either parasitic on primarily diurnal teleost fish and therefore must occupy their hosts' photic environment, or are swimming upstream to spawn in shallow rivers where, although they move at night, they must be exposed to relatively bright light while resting during the day. Hagfish, on the other hand, are benthic and apparently spend much of their time inside the carcasses of large dead fish on the bottom of the ocean at depths between 10 and 50 meters or more. Clearly, what is crucial to our argument is not nocturnality per se but the photic environment in which the organism under consideration has evolved.

The phylogenetic relationship between lampreys and hagfish has been the subject of some controversy but recent work using ribosomal RNA sequences suggests that they form a natural group (29). In discussing the difficulty involved in determining this relationship with certainty, Stock and Whitt (30) say "A further problem is the possibility that some proposed primitive features of hagfishes, especially those related to the eye (italics added), may actually be the result of more recent degenerative evolution linked to their burrowing habits and life at the ocean depths". We would argue that the photic environment associated with burrowing habits and life at ocean depths has selected for modification of the primitive cyclostome circadian organization (as represented by lampreys), which eliminated the pineal gland and other extraretinal circadian photoreceptors, leaving the eyes as sole source of photoreceptive input to the remaining circadian oscillators in the hypothalamus. Furthermore, we would point to the striking convergence of this pattern of circadian organization to that produced in early mammals by their presumed burrowing habits and life in the depths of the forest.

Of course it is difficult if not impossible to reconstruct enough of the photic environments of early cyclostome ancestors of lampreys and hagfish to be certain that the lamprey pattern is primitive and that of the hagfish degenerate. However, at least one prediction can be made and tested. If the hagfish pattern is degenerate we would predict that they would still retain melatonin-producing circadian oscillators in their retinas since this aspect of retinal organization seems always to be maintained (see below) and is therefore likely to be crucial for retinal function. If they do not synthesize retinal melatonin they may have diverged from the lineleading to lampreys before that hormone was invented by vertebrates, which would suggest that they never had a pineal gland, at least not one that synthesized melatonin and played the role that it has been shown to play in modern lampreys. Although the presence of retinal melatonin in hagfish would argue for their degeneracy and its absence for a circadian system evolved early and independently of the lamprey line, neither result would affect our conclusion that their circadian organization is the consequence of the low light intensities in their photic environment. 
Although the photic environment may exert strong selection pressure on circadian organization it is certainly not the only aspect of the environment to do so. Do we have any indication of how malleable vertebrate circadian organization is? The hagfish may have been benthic burrowers for a very long time and so cannot tell us much about the rate at which their circadian system has diverged from that of a lamprey-like ancestor (if indeed that is what has occurred). On the other hand, if the loss (or degeneration) of the pineal in owls is a result of their recently acquired nocturnality this would argue that such changes can occur reasonably rapidly.

We have described differences in circadian organization among three species of iguanid lizards (all diurnal but living in very different environments) and have hypothesized that the differences have been brought about by selection pressures exerted by their different environments over relatively short spans of time in geological terms (32). Recently, we have been studying a fourth species, Iguana iguana, and have found a somewhat different pattern of circadian organization. In these animals circadian oscillators exist in the retinas, the parietal eye and the pineal gland (19). Of these several melatonin-synthesizing organs only the pineal secretes melatonin into the bloodstream (33); however, the rhythm of circulating melatonin does not drive the locomotor rhythm in $I$. iguana as it does in birds (13), some other lizards (10), and probably lampreys (17). Pinealectomized iguanas retain robust rhythms of locomotor activity; however, they completely lose the equally robust (although low amplitude) internally generated rhythm of body temperature $(19,34)$. This pattern of circadian organization is clearly different from that of the two other iguanid lizards Anolis carolinensis and Sceloporus occidentalis (10) and perhaps from the pattern displayed by Dipsosaurus dorsalis $(32,35)$ although that has not been adequately tested. All of these lizards are diurnal and although each of them has multiple circadian photoreceptors and multiple circadian oscillators, the ways in which these are used to control particular behavioral or physiological rhythms are quite different. Although we do not know what selection pressures have produced these differences, their existence within a single lizard family argues for recent and rapid evolution.

One further example will serve to underline the malleability of the vertebrate circadian system. We have been measuring pineal and retinal rhythmicity at each of the three stages in the life cycle of Petromyzon marinus (see Figure 1). In the non-parasitic adult stage (preparatory to spawning) both the pineal and the retina of this organism synthesize melatonin rhythmically in vitro (19). Like L. japonica, their locomotor behavior is rhythmic in the field (36). The larvae of $P$. marinus are free living in shallow rivers for years before metamorphosis into an adult form parasitic on large teleosts. The larvae are conspicuously rhythmic, emerging from the mud each night to filter feed (37). Their pineals are also rhythmic in culture (19). The behavior of the parasitic form is quite different. After they have been feeding on a host fish for some time the fish either dies or shakes them off. They are active predators and immediately seek a new host using visual cues among others (36). Strict control of

\begin{tabular}{|l|c|c|c|}
\hline Life cycle & $\begin{array}{c}\text { Larvae } \\
\text { (4-5 years) }\end{array}$ & $\begin{array}{c}\text { Adult parasitic } \\
\text { (1.5-2 years) }\end{array}$ & $\begin{array}{c}\text { Adult non-parasitic } \\
\text { (5-6 months) }\end{array}$ \\
$\begin{array}{c}\text { Rhythmic } \\
\text { (nocturnal) }\end{array}$ & Non-rhythmic & $\begin{array}{c}\text { Rhythmic } \\
\text { (nocturnal) }\end{array}$ \\
Pineal melatonin & Rhythmic & Absent & Rhythmic \\
Retinal melatonin & Rhythmic & Rhythmic \\
\hline
\end{tabular}

Figure 1 - Schematic representation of the life cycle of the sea lamprey Petromyzon marinus. This cyclostome has a complex life cycle with three different phases: larval, adult parasitic, and adult non-parasitic. Melatonin is rhythmically synthesized by the pineal during the larval and non-parasitic adult stages, but not during the adult parasitic stage (see text). Retinal melatonin is synthesized rhythmically in both the parasitic and non-parasitic adult stages. In the larval stage the retina is not yet fully developed and so far we have not been able to culture it (compiled with data from Refs. 19,36,37). 
their locomotor behavior by circulating melatonin levels might handicap these animals in their search for a new host, particularly if melatonin played the same role as in the migrating or larval forms in which it may stimulate activity at night and/or suppress it during the day. It is therefore of great interest that the pineals of the parasitic stage of this lamprey do not synthesize melatonin rhythmically in vitro, in fact do not synthesize it at all (19). The assumption that the suppression of pineal melatonin synthesis in the parasitic stage is an adaptive ontogenetic modulation of circadian organization, is greatly strengthened by the observation that retinal melatonin synthesis is still rhythmic in these same parasitic animals (19). Everything that we know about retinal melatonin suggests that its primary function is to organize events within the retina. A visual predator like the parasitic lamprey obviously depends heavily on retinal function and so has maintained rhythmic melatonin synthesis in the retina while suppressing it in the pineal.

The examples that we have briefly described underline the fact that adjustment in circadian organization can be made over short spans of (evolutionary) time and even within the lifetimes of individuals. In one sense the fact that the basic vertebrate circadian axis has endured for 500 million years is a tribute to the success with which it can be modified to accommodate the demands of life in a great number of different environments.

\section{References}

1. Besharse JC \& luvone PM (1983). Circadian clock in Xenopus eye controlling retinal serotonin $\mathrm{N}$-acetyltransferase. Nature, 305: 133-135.

2. Pierce ME, Sheshberadaran $H$, Zhang Z, Fox LE, Applebury ML \& Takahashi JS (1993). Circadian regulation of iodopsin gene expression in embryonic photoreceptors in retinal cell culture. Neuron, 10: 579-584

3. Menaker M \& Tosini G (1996). Beyond Xenopus: Direct demonstration of retinal circadian oscillators in other vertebrates. Investigative Ophthalmology and Visual Science, 37: 66 (Abstract).

4. Tosini G \& Menaker M (1995). Melatonin release by cultured parietal eye, pineal and retina in Iguana iguana. Physiologist, 38: 25 (Abstract).

5. Tosini G \& Menaker M (1996). Circadian rhythms in cultured mammalian retina. Science, 272: 419-421.

6. Takahashi JS, Murakami N, Nikaido SS, Pratt BL \& Robertson LM (1989). The avian pineal, a vertebrate model system of the circadian oscillator: circadian regulation of circadian rhythms by light, second messengers, and macromolecular synthesis. Recent Progress in Hormone Research, 45: 279-348.
7. Underwood H (1990). The pineal and melatonin: Regulators of circadian function in lower vertebrates. Experientia, 46: 120128.

8. Klein D (1979). Circadian rhythms in the pineal gland. In: Krieger DT (Editor), Endocrine Rhythms. Raven Press, New York.

9. Ralph MR, Foster RG, Davis FC \& Menaker M (1990). Transplanted suprachiasmatic nucleus determines circadian period. Science, 247: 975-978.

10. Underwood $H$ (1992). Endogenous rhythms. In: Gans C \& Crews D (Editors), Biology of the Reptilia. Vol. 18. University of Chicago Press, Chicago.

11. Zimmerman N \& Menaker M (1979). The pineal gland: a pacemaker within the circadian system of the house sparrow. Proceedings of the National Academy of Sciences, USA, 76: 999-1003.

12. Murakami N, Nakamura H, Nishi R, Marumoto N \& Nasu T (1994). Comparison of circadian oscillation of melatonin release in pineal cells of house sparrow, pigeon and Japanese quail, using cell perfusion systems. Brain Research, 651: 209214.

13. Chabot CC \& Menaker M (1992). The effects of physiological cycles of infused melatonin on circadian rhythmicity in pigeons. Journal of Comparative Physiology $A, 170:$ 615-622.
14. Heighl S \& Gwinner E (1994). Periodic melatonin in the drinking water synchronizes circadian rhythms in sparrow. Naturwissenschaften, 81: 83-85.

15. Takahashi JS \& Menaker M (1982). Role of suprachiasmatic nuclei in the circadian system of the house sparrow, Passer domesticus. Journal of Neuroscience, 2: 815-828.

16. Underwood $H$, Barrett RK \& Siopes $T$ (1990). The quail's eye: a biological clock? Journal of Biological Rhythms, 5: 257-265.

17. Morita Y, Tabata M, Uchida K \& Samejima $M$ (1992). Pineal-dependent locomotor activity of lamprey, Lampetra japonica, measured in relation to LD cycle and circadian rhythmicity. Journal of Comparative Physiology A, 171: 555-562.

18. Samejima M, Tamotsu S, Uchida K, Hamada N \& Morita Y (1995). Function of the pineal organ and melatonin in the circadian organization of lamprey. Sixth Sapporo Symposium on Biological Rhythms, 30 (Abstract).

19. Menaker M \& Tosini G (1996). The evolution of vertebrate circadian system. In: Honma K \& Honma S (Editors). Circadian Organization and Oscillatory Coupling. Hokkaido University Press, Sapporo. 
20. Kabasawa H \& Ooka-Souda S (1989). Circadian rhythms in locomotor activity of the hagfish, Eptatretus burgeri (IV). The effect of eye-ablation. Zoological Science, 6: 135-139.

21. Foster RG, Grace MS, Provencio I, De Grip WJ \& Garcia-Fernandez JM (1994). Identification of vertebrate deep brain photoreceptors. Neuroscience and Biobehavioral Reviews, 18: 541-546.

22. Nelson RJ \& Zucker I (1981). Absence of extraocular photoreception in diurnal and nocturnal rodents exposed to direct sunlight. Comparative Biochemistry and Physiology, 69A: 145-148.

23. Giebultowicz JM, Bell RA \& Imberski RB (1988). Circadian rhythm of sperm movement in the male reproductive tract of the gipsy moth, Lymantria dispar. Journal of Insect Physiology, 34: 527-532.

24. Solessio E \& Engbretson GA (1993). Antagonistic chromatic mechanisms in photoreceptors of the parietal eye of lizards. Nature, 364: 442-445.

25. Cooper HM, Tessoneaud A, Caldani M, Locatelli A, Richard S \& Viguier-Martinez MC (1993). Morphology and distribution of retinal ganglion cells (RGC) projecting to the suprachiasmatic nucleus in the sheep. Society for Neuroscience $A b$ stracts, 19: 701.11.
26. Foster RG, Timmers AM \& De Grip WJ (1989). A comparison of some photoreceptor characteristics in the pineal and retina. II: The Djungarian hamster (Phodopus sungorus). Journal of Comparative Physiology A, 165: 565-572.

27. Quay WB (1979). The parietal eye-pineal complex. In: Gans C, Northcutt RG \& Ulinski P (Editors), Biology of the Reptilia. Vol. 9. Academic Press, London.

28. Menaker M \& Oksche A (1974). The avian pineal organ. In: Farner DS \& King JR (Editors), Avian Biology. Academic Press, New York.

29. Roberts S (1965). Photoreception and entrainment of cockroach activity rhythms. Science, 148: 958-960.

30. Stock DW \& Whitt GS (1992). Evidence from $18 \mathrm{~S}$ ribosomal RNA sequences that lampreys and hagfishes form a natural group. Science, 257: 787-789.

31. Ooka-Souda S, Kadota T \& Kabasawa H (1993). The preoptic nucleus: the probable location of the circadian pacemaker of the hagfish, Eptatretus burgeri. Neuroscience Letters, 164: 33-36.

32. Janik DS \& Menaker M (1990). Circadian locomotor rhythms in the desert iguana. I: The role of the eyes and the pineal. Journal of Comparative Physiology $A, 166$ : 803-810.
33. Tosini G \& Menaker M (1996). The pineal complex and melatonin affect the expression of the daily rhythm of behavioral thermoregulation in the green iguana. Journal of Comparative Physiology A, 179: 135142.

34. Tosini G \& Menaker M (1995). Circadian rhythm of body temperature in an ectotherm, Iguana iguana. Journal of Biological Rhythms, 10: 248-255.

35. Janik DS, Pickard GE \& Menaker M (1995). Circadian locomotor rhythms in the desert iguana. II: Effects of electrolytic lesions to the hypothalamus. Journal of Comparative Physiology A, 166: 811816.

36. Hardisty MW \& Potter IC (1971). The general biology of adult lampreys. In: Hardisty MW \& Potter IC (Editors), The Biology of Lampreys. Academic Press, London.

37. Hardisty MW \& Potter IC (1971). The behaviour, ecology and growth of larval lampreys. In: Hardisty MW \& Potter IC (Editors), The Biology of Lampreys. Academic Press, London. 